\title{
Dynamics of quantum entanglement
}

\author{
Karol Życzkowski ${ }^{1,2 *}$, Paweł Horodecki ${ }^{3},{ }^{* *}$, Michał Horodecki ${ }^{4}, * * *$, Ryszard Horodecki 4, , \\ ${ }^{1}$ Centrum Fizyki Teoretycznej PAN, Al. Lotników 32/46, 02-668 Warszawa, Poland \\ 2 Instytut Fizyki im. Smoluchowskiego, Uniwersytet Jagielloński, ul. Reymonta 4, 30-059 Kraków, Poland \\ ${ }^{3}$ Wydziat Fizyki Technicznej i Matematyki Stosowanej, Politechnika Gdańska, 80-952 Gdańsk, Poland \\ ${ }^{4}$ Instytut Fizyki Teoretycznej i Astrofizyki, Uniwersytet Gdański, 80-952 Gdańsk, Poland
}

\begin{abstract}
A model of discrete dynamics of entanglement of bipartite quantum state is considered. It involves a global unitary dynamics of the system and periodic actions of local bistochastic or decaying channel. For initially pure states the decay of entanglement is accompanied with an increase of von Neumann entropy of the system. We observe and discuss revivals of entanglement due to unitary interaction of subsystems. For some mixed states having different marginal entropies of the subsystems we find an asymmetry in speed of entanglement decay. The entanglement of these states decreases faster, if the depolarizing channel acts on the "classical" subsystem, characterized by smaller marginal entropy.
\end{abstract}

PACS numbers: 03.65 Bz, 03.67.-a

\section{INTRODUCTION}

Quantum entanglement is one of the most subtle and intriguing phenomena in nature [1,2]. Its potential usefulness has been demonstrated in various applications like quantum teleportation, quantum cryptography and quantum dense coding. On the other hand, quantum entanglement is a fragile feature, which can be destroyed by interaction with the environment. This effect due to decoherence [3], is the main obstacle for practical implementation of quantum computing. A model allowing to study the dynamics of entanglement in presence of interaction with the environment has been recently analysed by Yi and Sun 4 .

In this paper we investigate destruction of the entanglement in a proposed model of discrete dynamics. We consider a simple bipartite system consisting of two spin$\frac{1}{2}$ particles. Only one of them is subjected to periodic actions of a quantum channel, which represents the interaction with environment. As the initial states we choose random states taken from the ensemble of pure separable states and from the ensemble of maximally entangled pure states. We also investigate the time evolution of mixed states having some special property. The corresponding system is composed of two subsystems exhibiting different properties with respect to some entropy inequality which is satisfied by all classical systems. One of the subsystems satisfies the inequality and may be considered "classical", while the other, "quantum" subsystem violates the inequality. We investigate an asymmetry in the process of destruction of entanglement with respect to the subsystem interacting with the environ- ment. We demonstrate a possible presence of revivals of entanglement caused by the global unitary evolution entangling the subsystems between consecutive actions of the environment.

The paper is organised as follows. In section II we describe a simple model of discrete time evolution. In section III we derive bounds on the entropy increase under the action of the environment. Then in section IV we analyse the decrease of entanglement versus increase of the degree of mixing of the initially pure states. The asymmetry in the entanglement decay depending on the subsystem subjecting to influence of environment is described in section V. In section VI we consider the entanglement revivals. The results of the paper are discussed in section VII.

\section{MODELS OF TIME EVOLUTION}

In this paper we consider the bipartite state subjected sequential interactions with environment. They are modelled by quantum channels, defined as completely positive linear maps, preserving the trace of the state [5].

Let $\sigma$ be a density operator acting on a finitedimensional Hilbert space $\mathcal{H}$. The most general form of the quantum channel is the following transformation $\sigma \rightarrow \sigma^{\prime}$ :

$$
\sigma^{\prime} \equiv \Lambda(\sigma)=\sum_{i=1}^{K} V_{i} \sigma V_{i}^{\dagger}, \quad \text { where } \quad \sum_{i=1}^{K} V_{i}^{\dagger} V_{i}=\mathbb{I} .
$$

If in addition $\sum_{i=1}^{K} V_{i} V_{i}^{\dagger}=\mathbb{I}$ holds then the channel is called bistochastic.

Bistochastic channels can be alternatively defined as channels which do not decrease von Neumann entropy of quantum states.

A particular example of the bistochastic channel is given by random external fields [6]. They can be written as

$$
\sigma^{\prime} \equiv \Lambda_{R}(\sigma)=\sum_{i=1}^{K} p_{i} A_{i} \sigma A_{i}^{\dagger}
$$

where $A_{i}, i=1,2, \ldots, K$ are unitary operators and the vector of probabilities $\vec{p}=\left[p_{1}, \ldots, p_{K}\right]$ is normalised

$$
\sum_{i=1}^{K} p_{i}=1, \quad p_{i} \geq 0
$$


Such random systems can be described in the formalism of quantum iterated function systems [7]. The so called Kraus form (11) can be reproduced setting $V_{i}=\sqrt{p_{i}} A_{i}$. It is worth to note that in the case of the most elementary quantum system described on the Hilbert space $\mathcal{H}=\mathcal{C}^{2}$ the channel is bistochastic if and only if it is a random external field (2) (see [8]). Note that an unitary evolution of the system can be considered as the simplest case of the bistochastic quantum channel with $K=1$.

There exist, however, many quantum channels which are not bistochastic. We shall consider the following decaying channel, sometimes called [9] the amplitude damping channel)

$$
\sigma^{\prime} \equiv \Lambda_{D}(\sigma)=M_{1} \sigma M_{1}+M_{2} \sigma M_{2}
$$

where the matrices $M_{1}=\left[\begin{array}{cc}1 & 0 \\ 0 & \sqrt{p}\end{array}\right]$ and $M_{2}=$ $\left[\begin{array}{rr}0 & \sqrt{1-p} \\ 0 & 0\end{array}\right]$ are written in the standard basis.

Let $\varrho$ denote a mixed state of a $2 \otimes 2$ system i. e. the density operator defined on the Hilbert space $\mathcal{H}=\mathcal{H}_{A} \otimes \mathcal{H}_{B}=\mathcal{C}^{2} \otimes \mathcal{C}^{2}$. The system consists of two subsystems $A$ and $B$ which can represent spin- $\frac{1}{2}$ particles or two-level atoms. In our model the unitary dynamics is interrupted by periodic actions of the environment as shown schematically in Fig. 1.

Discrete time evolution of the state $\varrho$ reads in our model

$$
\varrho(n+1)=U \varrho^{\prime}(n) U^{\dagger}=U\left(\hat{\Lambda}(\varrho(n)) U^{\dagger}\right.
$$

where $\hat{\Lambda}=I \otimes \Lambda$ and the channel $\Lambda$ is either bistochastic (2) or decaying (1). Here $U=e^{i \alpha \tilde{H}}$ represents a unitary transformation which involves an interaction between both subsystems $A$ and $B$ described by the Hamiltonian $\tilde{H}$. We use the dimensionless units and $\alpha$ stands for a coupling parameter. Subsequently we shall consider the cases with $\tilde{H}$ equal either to $\sigma_{x} \otimes \sigma_{y} \equiv H$ or to $\tilde{H}=\sigma_{y} \otimes \sigma_{x} \equiv H^{\prime}$.

In general we shall use four types of dynamics defined by four different operators $\Lambda$-s in the formula (5). Three of them will be random external fields $\Lambda_{R}(2)$, all defined by the same set of $K=4$ unitary operators: $A_{1}=\mathbb{I}$, $A_{2}=\sigma_{1}, A_{3}=\sigma_{2}, A_{4}=\sigma_{3}$ (where $\sigma_{i}$ denote Pauli matrices), but with different vectors of probability: (3):

$$
\begin{aligned}
\vec{p}^{(1)} & =[1-\epsilon, 0,0, \epsilon] \\
\vec{p}^{(2)} & =\left[1-\epsilon, 0, \frac{\epsilon}{2}, \frac{\epsilon}{2}\right] \\
\vec{p}^{(3)} & =\left[1-\epsilon, \frac{\epsilon}{3}, \frac{\epsilon}{3}, \frac{\epsilon}{3}\right], 0 \leq \epsilon \leq 1 .
\end{aligned}
$$

Each dynamics depends on two continuous parameters: $\alpha$ contained in $U=e^{i \alpha \tilde{H}}$ governing the unitary dynamics and $\epsilon$, included in the vector of probabilities, and describing the strength of the coupling with the environment. Additional discrete index $j$ labels the different vectors of probability, $\vec{p}^{(j)}$. For these three models of dynamics we shall use the compact notation $\Theta_{\alpha, \epsilon}^{j}$. The fourth dynamics denoted by $\Theta_{\alpha, p}$ is defined by putting in formula (5) the decaying channel (田). Dynamics involving the operation $U$ with "reflected" (i. e. obtained from $H$ by permutating subsystems) Hamiltonian $H^{\prime}=\sigma_{y} \otimes \sigma_{x}$ will be denoted by the same symbols with only one change: $\Theta \rightarrow \tilde{\Theta}$.

Remark.- If $\alpha$ is equal to zero, then the unitary operation $U$ in (5) is reduced to identity transformation. In particular, it can be seen that the dynamics $\Theta_{0, \epsilon}^{3}$ corresponds to periodic action of depolarizing channel [11].

Now the essence of our study is the following: we consider composite quantum systems subjected to the local interaction with the environment, which acts on one subsystem only. We investigate, how the decay of the entanglement in the system depends on the initial state and the type of the dynamics. In particular we analyse, to which extent the decrease of the mean entanglement is reflected by the evolution of von Neumann entropy of the system.

\section{BOUNDS ON ENTROPY INCREASE UNDER LOCAL CHANNEL}

We start establishing bounds for the increase of von Neumann entropy.

Proposition .- Under a local action of the quantum channel $\varrho_{A B} \rightarrow(\mathbb{I} \otimes \Lambda) \varrho_{A B}$, the increase of the von Neumann entropy $\Delta S$ for a bipartite $n \otimes m$ state is bounded by

$$
\Delta S \equiv S\left(\varrho_{A B}^{\text {out }}\right)-S\left(\varrho_{A B}^{\text {in }}\right) \leq S\left(\varrho_{A}^{\text {in }}\right)-S\left(\varrho_{A B}^{\text {in }}\right)+\log m,
$$

where $S\left(\varrho_{A}\right)$ denotes the entropy of the subsystem $A$. In particular, if the system is separable then $\Delta S \leq \log m$.

Proof.- By the definition the local channel is trace preserving, hence it does not change the density matrix of the first subsystem. Thus $\varrho_{A}^{\text {out }}=\varrho_{A}^{\text {in }}$ and the same holds for the corresponding entropies. Then from subadditivity of the entropy we have

$$
\begin{array}{r}
S\left(\varrho_{A B}^{\text {out }}\right) \leq S\left(\varrho_{A}^{\text {out }}\right)+S\left(\varrho_{B}^{\text {out }}\right)=S\left(\varrho_{A}^{\text {in }}\right)+S\left(\varrho_{B}^{\text {out }}\right) \leq \\
S\left(\varrho_{A}^{\text {in }}\right)+S_{\max }^{B} \equiv S\left(\varrho_{A}^{\text {in }}\right)+\log m .
\end{array}
$$

We get the first inequality in the Proposition by subtracting $S\left(\varrho_{A B}^{i n}\right)$ from both sides of the above inequality. For separable states one always has $S\left(\varrho_{A B}^{i n}\right)-S\left(\varrho_{A}^{i n}\right) \geq 0$ 12] which simplifies (7) to $\Delta S \leq \log m$ as expected.

Note that a sequence of quantum channels acting locally forms a quantum channel acting locally too. So the proposition works also for the dynamics $\Theta_{0, \epsilon}^{i}$ and $\Theta_{0, p}$. Moreover, from Eq. (河) we see that the entropy of initially pure separable state $\varrho_{A B}^{i n}$ cannot exceed $\log m$. 


\section{ENTANGLEMENT VERSUS DEGREE OF MIXING}

In this section we study the time evolution of entanglement and compare it with the time evolution of von Neumann entropy. To characterise the degree of entanglement we use the entanglement of formation introduced by Bennett et al. [11]. For any $2 \otimes 2$ mixed state this quantity may be computed analytically as shown by Hill and Wootters 13. In this case the entanglement of formation $E$ (or shorter, the entanglement) varies from zero (separable states) to $\ln 2$ (maximally entangled states), so in the figures we used the rescaled variable $E / \ln 2$.

Our results were obtained by averaging over ensembles of random initial states. They were generated according to natural measures on:

(i) 6 dimensional manifold of all pure states for $2 \otimes 2$ problem,

(ii) 3 dimensional manifold of maximally entangled pure states,

(iii) 4 dimensional manifold of separable pure states.

Numerical experiments have shown that the samples of 100 initial states, generated randomly as described in the appendix, were sufficient to receive reliable results.

\section{A. Bistochastic channels}

As shown in 14, 15 the mean entanglement of mixed states decreases monotonically with increasing degree of mixing. Due to interaction with the environment the initially pure states become mixed: their von Neumann entropy, $S(\varrho)=-\operatorname{Tr}(\varrho \ln \varrho)$, grows in time. Thus it is natural expect a corresponding monotonous decay of the mean entanglement. This indeed takes place, as shown in Fig. 2 in absence of the unitary dynamics, $(\alpha=0)$. Initial states were taken randomly from the entire space of pure states, so in accordance with 15, the initial mean entanglement is close to $(\ln 2) / 2$. The parallel processes of decay of the entanglement and increase of the entropy are accelerated, if the parameter $\epsilon$ describing the interaction with environment increases.

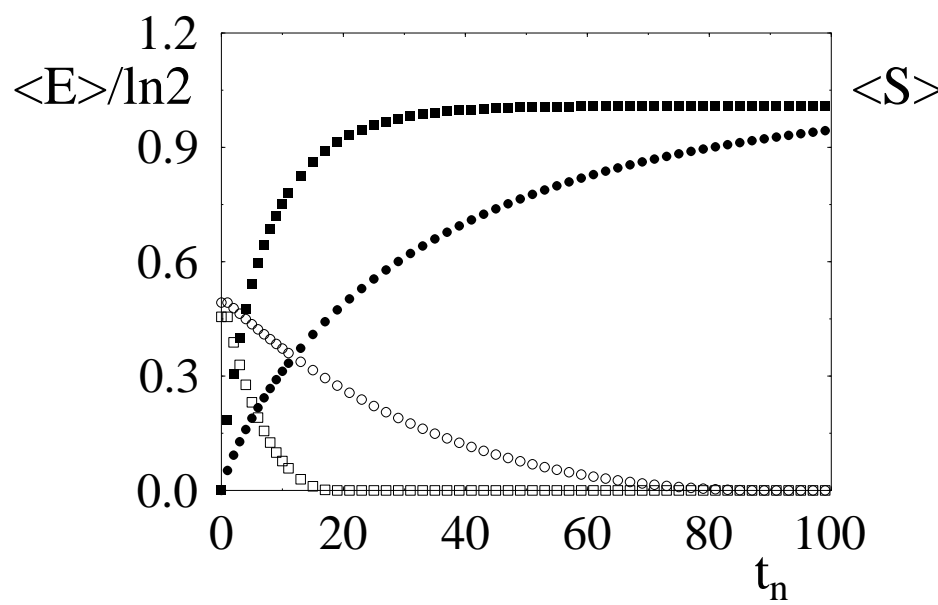

FIG. 2. Dynamics of quantum entanglement for system $\Theta_{0, \epsilon}^{2}$. Mean entanglement of formation $\langle E\rangle$ (open symbols) and von Neumann entropy $\langle S\rangle$ (closed symbols) averaged over a sample of 100 random pure states shown as functions of discrete time $t_{n}$. No unitary evolution is present, $(\alpha=0)$. Parameter $\varepsilon$, controlling the interaction with environment is set to $0.01(\circ)$ or $0.05(\square)$.

For initially maximally entangled pure states (case (ii)) a similar dependence is represented by circles in Fig. 3. Here $\langle E(0)\rangle=\ln 2$. The picture changes when unitary evolution is involved. The latter leads to oscillations of entanglement of formation, reflected in the time evolution of entropy. The frequency of oscillations is proportional to $\alpha$. The larger this parameter, the faster the unitary evolution $U$ rotates the states $\varrho$ from and into the convex set of separable states. In the case of entropy, oscillations are only due to changes of the second derivative i.e. entropy is still monotonically decreasing. This is not the case for entanglement $E$, which can also be seen in Fig. 4 for several individual initial states (without averaging). For short times the curve for $\alpha=0$ (no unitary evolution) seems to constitute an envelope for all other curves. 

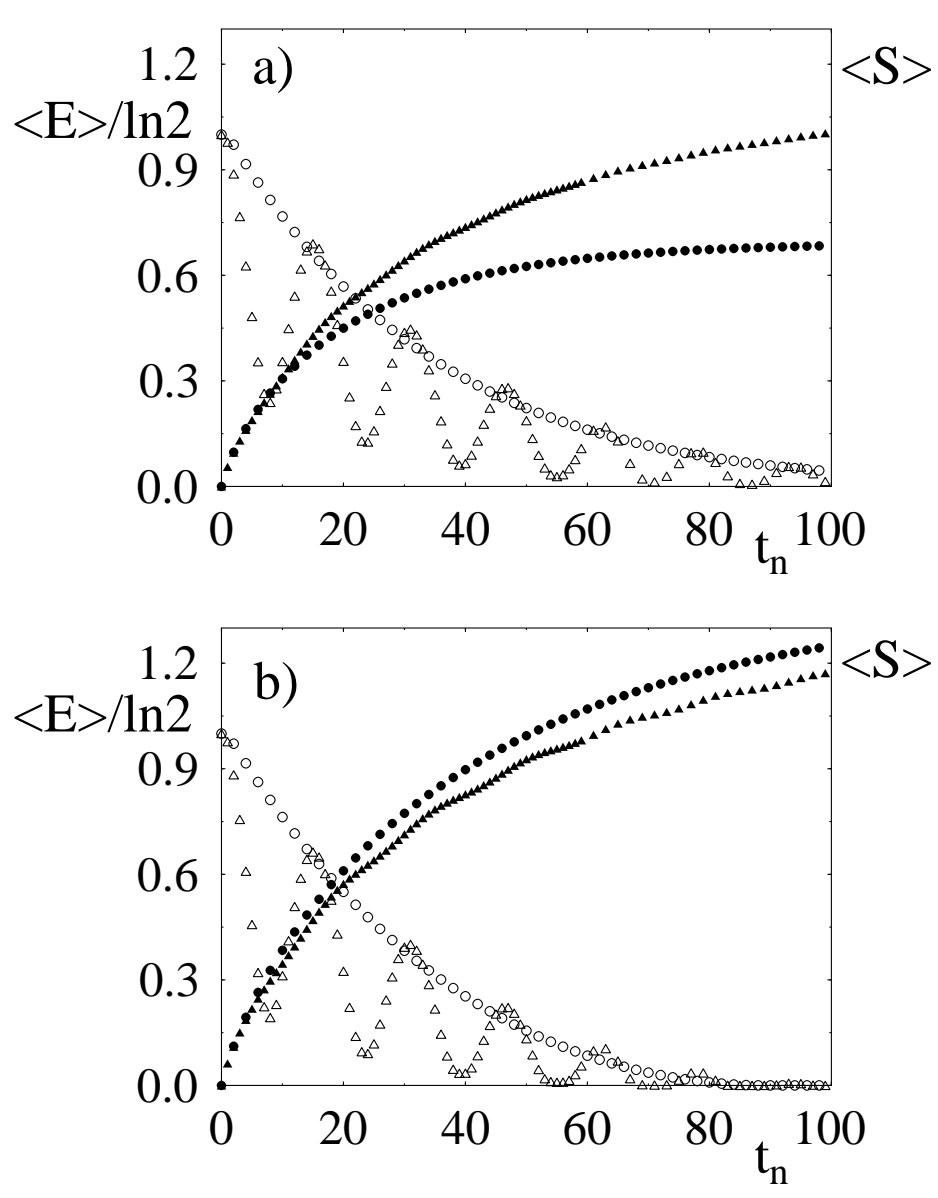

FIG. 3. As in Fig. 2 for a sample of 100 maximally entangled states $(E(0)=\ln (2))$ with $\varepsilon=0.01 ; \alpha=0.0(\circ)$ and $\alpha=0.1(\triangle)$ for channels described by a) $\vec{p}^{(1)}$ and b) $\vec{p}^{(2)}$. Observe how the influence of the unitary dynamics depends on the kind of the channel.

It is worth to emphasise a significant difference between $\Theta_{\alpha, \epsilon}^{1}$ (Fig. 3.a) and $\Theta_{\alpha, \epsilon}^{2}$ (Fig. 3.b). In the former case the presence of unitary evolution can accelerate the process of entropy increase. In the latter, on the contrary, switching on unitary evolution results in slower increase of the mean entropy. The iteration of the channel $\Theta_{\alpha, \epsilon}^{1}$ preserves both the number and the position of the nonzero component in $\vec{p}$. It is not the case for $\Theta_{\alpha, \epsilon}^{2}$, for which two Pauli matrices generate the entire algebra of unitary matrices $A_{i}$ involved.

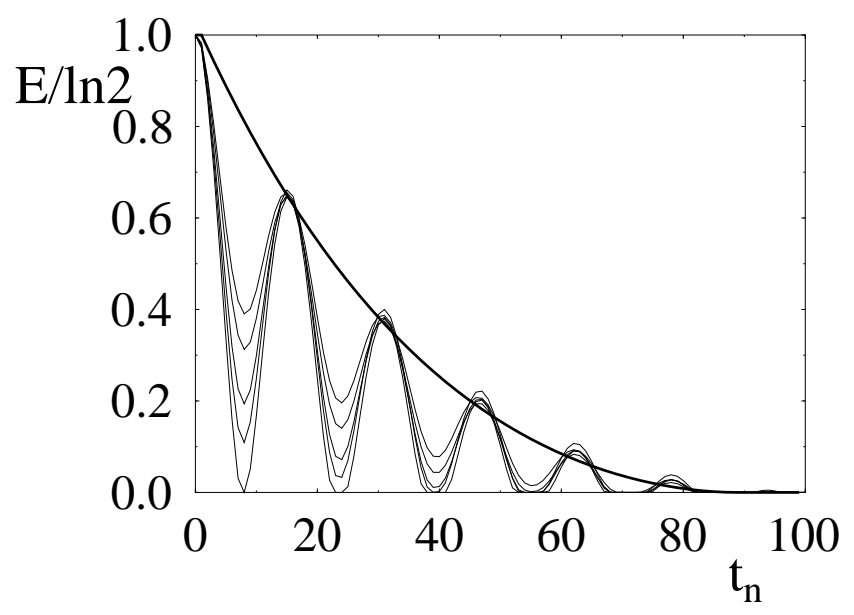

FIG. 4. Dependence of entanglement of formation on time for several randomly chosen maximally entangled pure states. The unitary dynamics $U=\exp (i \alpha \tilde{H})$ is governed by the parameter $\alpha$. Here $\varepsilon=0.01$ in $\vec{p}^{(2)}$, and $\alpha=0.1$ (narrow lines). Reference bold line represents the case of no unitary dynamics $(\alpha=0)$, for which the dynamics of entanglement does not depend on the initial state.

Consider now the case (iii), of initially separable states, presented in Fig. 5. The presence of the unitary evolution may increase the mean entanglement, initially equal to zero. However, there is one difference more; for both dynamics $\Theta_{\alpha, \epsilon}^{1}$ (Fig. 5.a) and $\Theta_{\alpha, \epsilon}^{2}$ (Fig. 5.b) presence of the unitary dynamics accelerates the process of increase of entropy. In absence of the unitary dynamics $(\alpha=0)$ the entropy does not exceed the value $\ln 2$, in accordance to our proposition proved in section III.

Obtained results show that the oscillations of the mean entanglement $E$ are anti-correlated with the oscillations of the entropy $S$. It was also checked that if $\alpha$ is kept constant, but the Hamiltonian is chosen randomly then the oscillations of entanglement are smeared out. It means that effects of quantum coherence are destroyed and the destruction of entanglement occurs faster. 


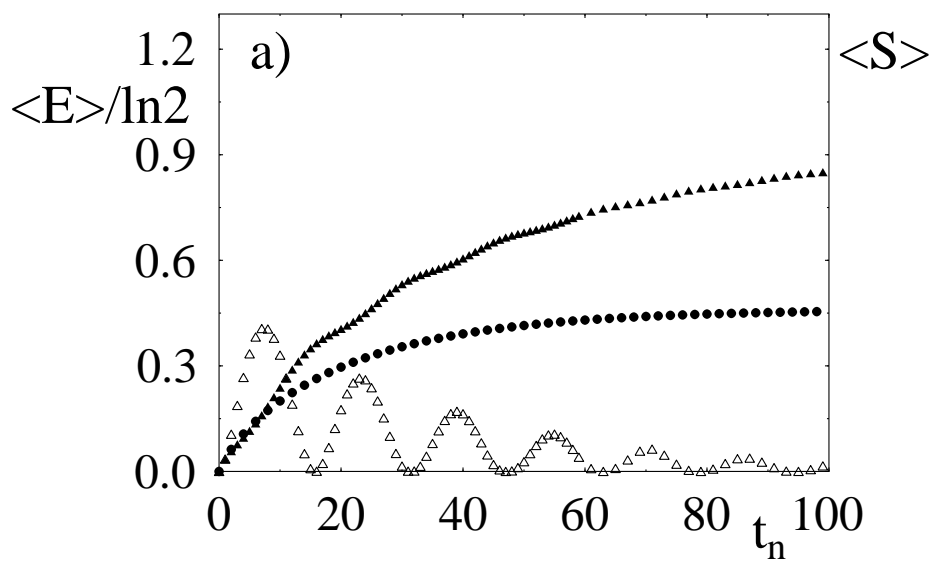

dumped to the ground state. So finally the state of the system is a product one consisting of the ground state of the affected subsystem and the reduced density matrix of the unperturbed subsystem. Thus, after the averaging procedure, one gets the averaged von Neumann entropy of the subsystem not subjected to action of the channel.

A random choice of initially pure states of the composite system induces a certain measure in the space of the reduced density matrices 16. As proved recently by Hall [17] the natural rotationally invariant measure on the space of $N=4$ pure states induces a uniform measure in the Bloch ball representing the density matrices for $N=2$. Denoting the spectrum of reduced matrices by $\{1 / 2-r, 1 / 2+r\}$ we may write more formally, $P(r)=24 r^{2}$ for $r \in[0,1 / 2]$. The von Neumann entropy, averaged over this measure equals $1 / 3$ [17], in agreement with the numerical data presented in Fig.6.
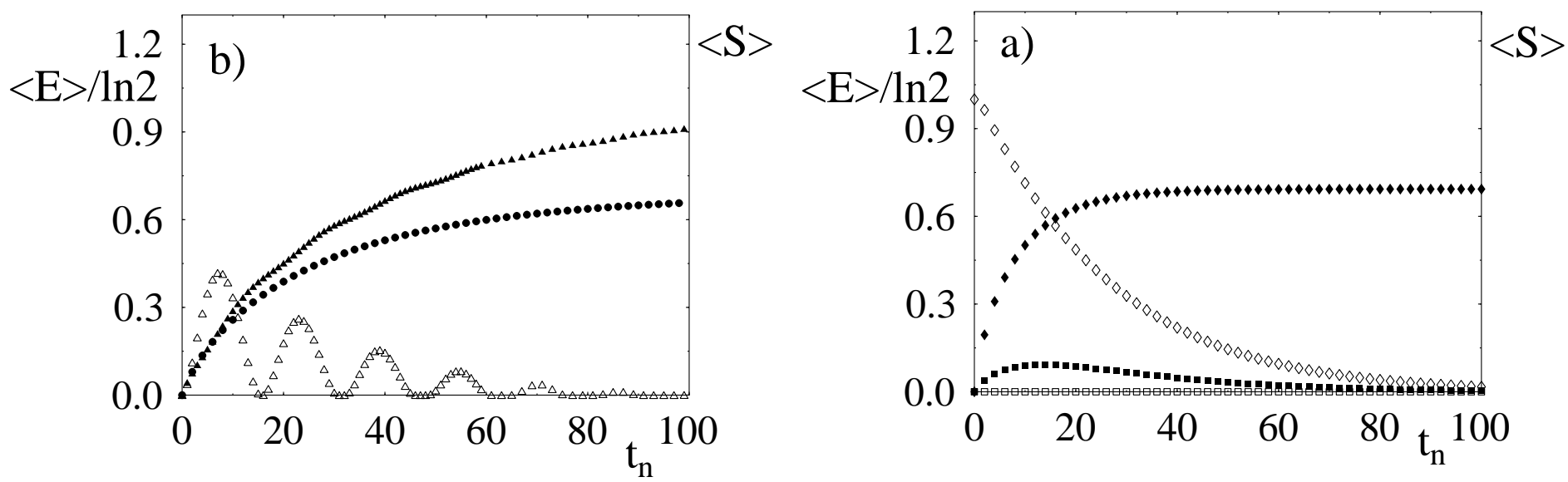

FIG. 5. As in Fig. 3 for a sample of 100 initially separable pure states $(E(0)=0)$. In absence of unitary dynamics, $(\alpha=0)$, the entanglement equals to zero.

\section{B. Decaying channel}

Figure 6 presents results obtained for the amplitude damping channel (4). In the absence of the unitary evolution $(\alpha=0)$ the mean entropy, $\langle S\rangle$, averaged over the entire manifold of pure states (case (i)), does not tend monotonically to its maximal value. At $t_{n} \sim 20$ the entropy reaches its maximum and then decreases to its limiting value about 0.3 (see full circles in Fig. 6b). This is due to the fact that for the decaying channel the entropy of the system may decrease.

Numerical data received by averaging over the set of maximally mixed states (case (ii), diamonds) and the set of separable pure states (case (iii), squares) are shown in Fig. 6a. Observe that the steady state limiting values of the von Neumann entropy, $\langle S\rangle$, represents the initial average entanglement $\langle E\rangle$. Indeed, in absence of the unitary evolution the perturbed subsystem is eventually

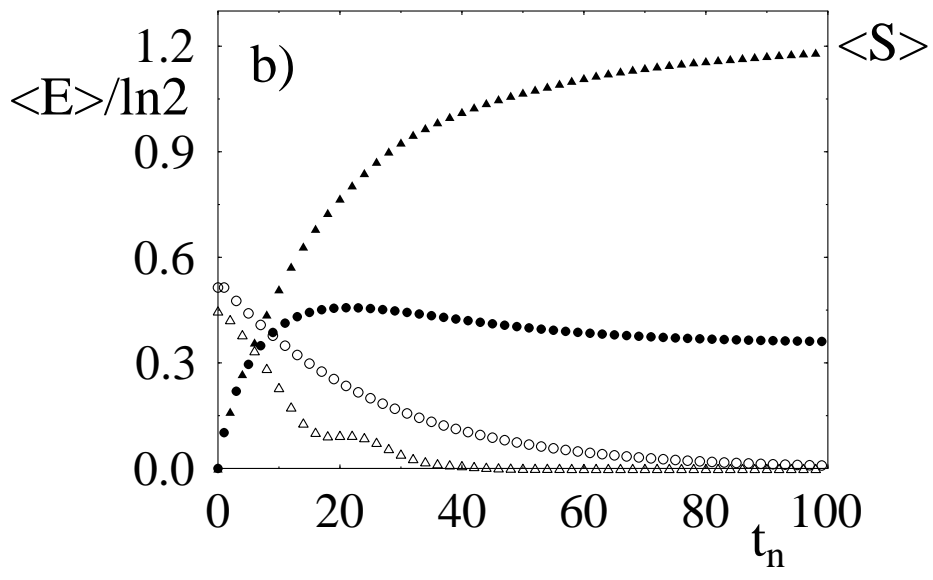

FIG. 6. As in Fig. 2 for samples of 100 pure states subjected to the Kraus channel (4) with $p=0.05$ and $\alpha=0.0$. Initial states drown randomly from the ensembles of a) ( $\square$ ) - separable pure states; a) $(\diamond)$ - maximally entangled pure states, and b) (o) - ensemble of all pure states. Case (i) with unitary evolution, $\alpha=0.1$, is denoted by $(\triangle)$ in panel b).

For non-zero values of $\alpha$ we observe the oscillations of the mean entanglement, caused by the unitary evo- 
lution. It is interesting, however, that the presence of unitary evolution allows the final entropy to be maximal (see full triangles in Fig. 6b). It means that the presence of the decay channel is completely masked by the unitary interaction between both subsystems.

\section{ASYMMETRY OF ENTANGLEMENT DECAY}

We shall consider here dynamics of mixed states having an intriguing property. Namely we choose a quantum bipartite system, which violates some entropy inequality only with respect to one of both subsystems. Let us recall first that the information gain resulting from the measurement of any of subsystems of a quantum state with classical correlations is not greater than the gain obtain form measurement performed on the entire system. This classical feature is characteristic of quantum separable states. They do satisfy the following two inequalities concerning von Neumann entropy [12,18]:

$$
S\left(\varrho_{A B}\right) \geq S\left(\varrho_{A}\right)
$$

and

$$
S\left(\varrho_{A B}\right) \geq S\left(\varrho_{B}\right)
$$

where $\varrho_{A}$ and $\varrho_{B}$ denote the reduced density matrices, e.g. $\varrho_{A} \equiv \operatorname{Tr}_{B}\left(\varrho_{A B}\right)$. Now we shall focus on the following family of states introduced in 19]. They can be written as $\varrho^{(1)}:=q\left|\Psi_{1}\right\rangle\left\langle\Psi_{1}|+(1-q)| \Psi_{2}\right\rangle\left\langle\Psi_{2}\right|, 0<q<1$, with normalised pure state vectors $\left|\Psi_{1}\right\rangle=a|00\rangle+\sqrt{1-a^{2}}|11\rangle$ and $\left|\Psi_{2}\right\rangle=a|10\rangle+\sqrt{1-a^{2}}|01\rangle$ with $0<a<1$. In the standard basis, $(|00\rangle,|01\rangle,|10\rangle,|11\rangle)$, the corresponding density matrix takes the form

$$
\varrho^{(1)}=\left[\begin{array}{ccc}
q a^{2} & 0 & 0 \\
0 & (1-q)\left(1-a^{2}\right) & (1-q) a \sqrt{1-a^{2}} \\
0 & (1-q) a \sqrt{1-a^{2}} & (1-q) a^{2} \\
q a \sqrt{1-a^{2}} & 0 & 0
\end{array}\right.
$$

Let us take $a^{2}>q>\frac{1}{2}$. Then the first inequality (9) is violated, while the second one $(10)$ is not. Thus the composite system can be called 'quantum' with respect to the subsystem $A$ and 'classical' with respect to the subsystem $B$. One may then expect that the bipartite system will loose entanglement in different ways, depending on whether the environment interacts with classical or quantum subsystem. Intuitively one could guess that the entanglement should be more robust if the noise affects the classical subsystem.

Here we studied the system $\rho^{(1)}$ for $q=3 / 5$ and $a^{2}=3 / 4$. Then von Neumann entropy of the entire system, $S=s(2 / 5) \approx 0.673$ is greater than the entropy of the classical subsystem $B$ for which $S_{B}=s(1 / 4) \approx 0.562$, and smaller than the entropy of the quantum subsystem, $S_{A}=s(9 / 20) \approx 0.688$, where $s$ stands for the binary
Shannon entropy, $s(x):=-x \ln x-(1-x) \ln (1-x)$. We analysed the time evolution of this quantum system in presence of a depolarizing channel $\Theta_{3, \epsilon}^{0}$ given by (2). In the theory of error correcting codes it is one of the most popular models of environment induced noise. The evolution of entanglement for the state $\varrho^{(1)}$ is represented by stars in Fig.7. In this case the bistochastic channel $\tilde{\Lambda}$ acts on the 'classical' subsystem $B$. To investigate a possible asymmetry of the entanglement decay we consider the state $\rho^{(2)}$, for which both subsystems are exchanged. More precisely, all elements of both density matrices are equal, apart from $\rho_{23}^{(2)}=\rho_{32}^{(1)}$ and $\rho_{32}^{(2)}=\rho_{23}^{(1)}$. The corresponding dynamics of $\rho^{(2)}$ is denoted by crosses in Fig. 7. In this case the noise interacts with the 'quantum' subsystem $A$. The magnification in the inset reveals the asymmetry in the time evolution. Observe that the attack on the 'classical' part of the system is more harmful to the entanglement properties of the system. This counter intuitive effect, called subsequently anomalous entanglement decay (AED), links quantum and classical features of the state from information-theoretical point of view.

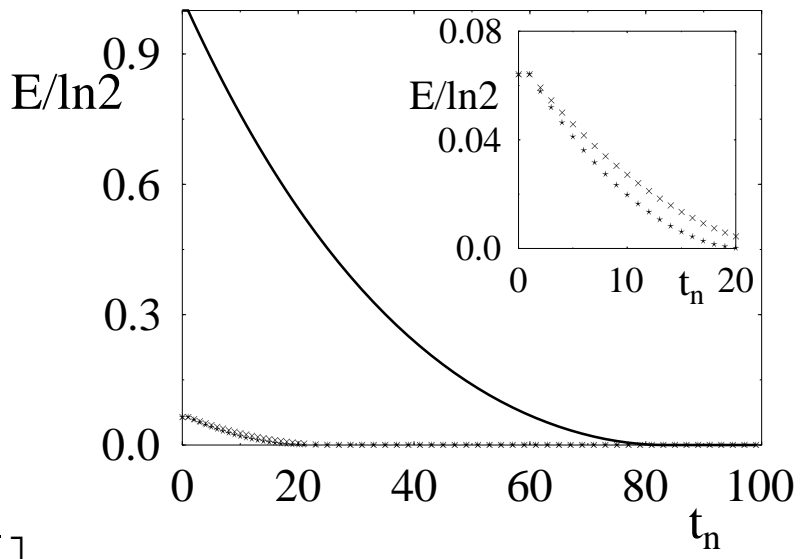

FIG. 7. Comparison of the dependence of the entanglement of formation for the state $\varrho^{(1)}$ with $a^{2}=3 / 4 ; q=3 / 5(\star)$ and $\varrho^{(2)}(\times)$. The bistochastic channel $\vec{p}_{3}$ with $\varepsilon=0.01$ teracts with the 'classical' subsystem $B$ in the former case, and with the 'quantum' subsystem $A$ in the latter case. Solid line represents the behaviour of a maximally entangled state $\rho_{\text {max }}$. Magnification of the initial dependence provided in the inset reveals the asymmetry of the entanglement decay.

Let us recall that any $2 \otimes 2$ system may be described by two Bloch vectors, representing locally both subsystems, and a correlation matrix $T$, which represents the projection of the composite system onto the family of mixtures of maximally entangled states (see [20]). A possible explanation of AED should take into account the fact that the local action of environment changes both the Bloch vectors, the correlation matrix, as well as their relationship. A depolarizing channel may affect in a similar way both local parameters, but it may distinguish, (in sense of the destruction of the entanglement), the correlation parameters with respect to the side of the action. 
It should be noted that, regardless which part is subjected to the noise, the entanglement of mixed states $\rho^{(1)}$ and $\rho^{(2)}$ decreases slower than the entanglement of the maximally entangled states (bold line in Fig. 7). This is due to the fact that the latter decreases fast for short times and slow at longer time scales, for which the initially pure state gets mixed. It is thus instructive to compare the shape of the bold line starting from $t_{N} \approx 60$ with the symbols representing the initial decay of entanglement of the states $\rho^{(i)}$.

\section{AMPLIFYING THE PROCESSES: ENTANGLEMENT REVIVALS.}

Consider now the depolarizing dynamics $\Theta_{\alpha, \epsilon}^{3}$ with an unitary operation involved, $(\alpha \neq 0)$, affecting either subsystems $A$ or $B$. To compare the dynamics of both symmetric mixed states $\varrho^{(1)}$ and $\varrho^{(2)}$ we study their unitary interaction governed by the Hamiltonians: $H=\sigma_{x} \otimes \sigma_{y}$, and the reflected one $H^{\prime}=\sigma_{y} \otimes \sigma_{x}$.
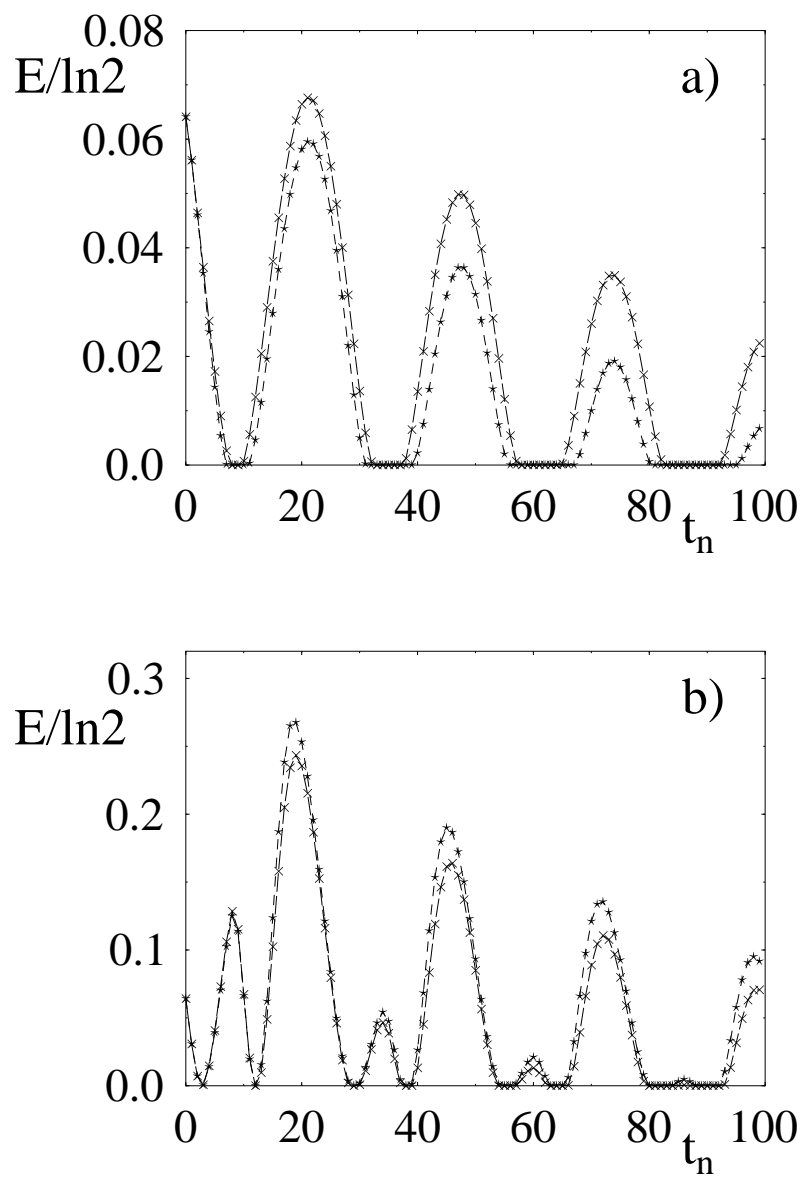

FIG. 8. Unitary dynamics and asymmetry of entanglement decay: a) the state $\varrho^{(1)}$ defined by parameters $a^{2}=3 / 4$ and $q=3 / 5$ subjected to the bistochastic channel $\vec{p}_{3}$ with $\varepsilon=0.002$ and unitary dynamics $H$ with $\alpha=0.06(\star)$; the symmetric state $\varrho^{(2)}$ interacting with the reflected Hamiltonian $H^{\prime}(\times)$. Panel b) shows the data for reflected unitary dynamics; the Hamiltonians $H^{\prime}$ and $H$ are exchanged.
Let us consider two cases:

(a) the noise parameter $\epsilon$ is much less than the parameter $\alpha$ characterising the unitary interaction,

(b) both parameters are of the same order of magnitude.

Numerical results obtained in the weak noise case (a) are presented in Fig. 8. and 9. The revivals of the entanglement, caused by the unitary interaction, are manifestly visible, since the strength of the interaction with the environment $\epsilon=0.002$ is much less then the parameter $\alpha=0.06$ governing the unitary dynamics. Note the characteristic entanglement plateaus, if the analysed state travels across the set of the separable states and the entanglement attains its minimal value equal to zero. The effect of anomalous entanglement decay is clearly visible in Fig. 8.a, where the entanglement decays faster if the environment interacts with the classical subsystem. This contrasts the situation shown in Fig. 8.b, for which the unitary evolution is due to the reflected Hamiltonian $H^{\prime}$ and the exposure of the 'quantum' subsystem to the action of the environment action is more damaging for the entanglement.
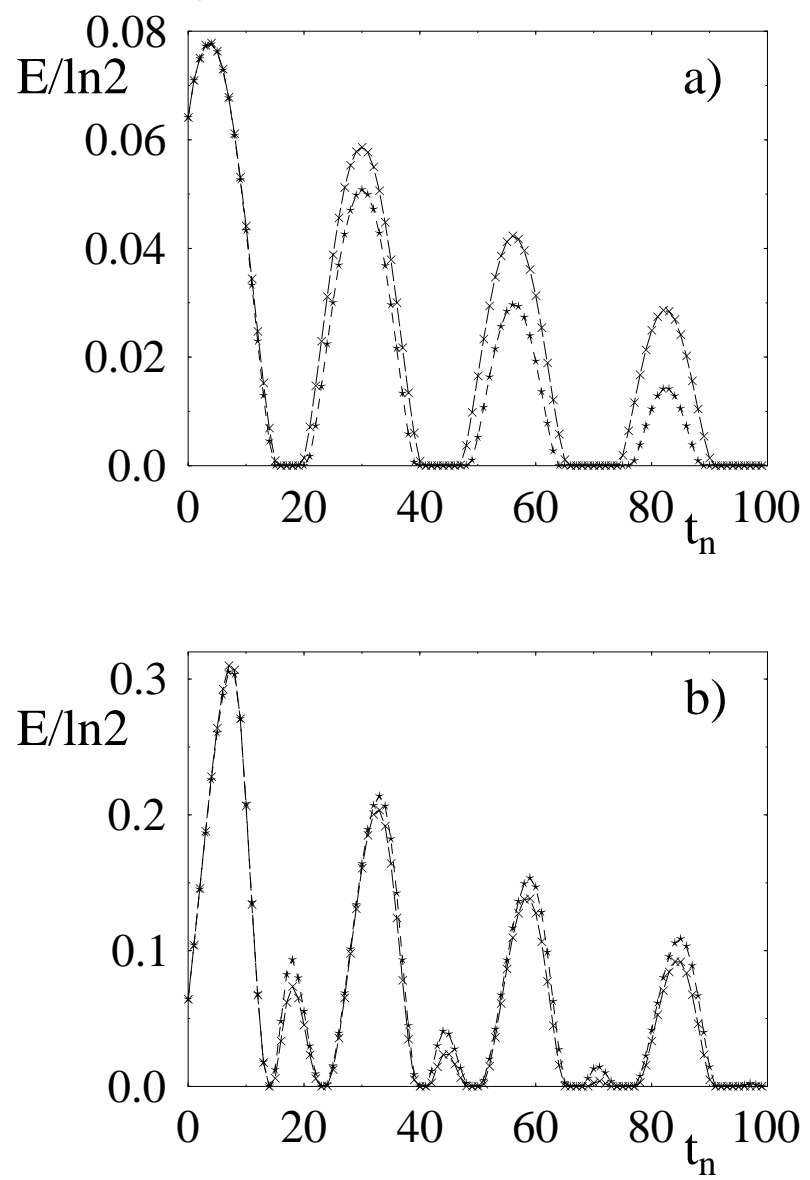

FIG. 9. As in Fig. 8 for $\varepsilon=0.002$ and $\alpha=-0.06$, i. e. the process runs back in time. Observe that maxima in Fig. 8.a correspond to minima in Fig. 9.a and vice versa.

It is instructive to analyse the same system with the unitary evolution reversed in time. Such a case, obtained 
by a change of the parameter $\alpha \rightarrow-\alpha$, is presented in Fig. 9. The general character of the evolution is kept. The significant difference is that here the entanglement is amplified at the very beginning which may have practical consequences if we are interested in short times of the process. Note that the figures 8.a. and 9.a reflected along the vertical line at $t_{n}=0$ (respectively, 8.b and reflected 9.b) exhibit some kind of symmetry with respect to the initial moment.

What happens if we allow the strength of the coupling with the environment to be comparable with the parameter of the unitary interaction? This situation, corresponding to the case (b), is illustrated in Fig. 10. Here some interesting qualitative changes occur. The AED effect is present in the case shown in Fig. 10a; at the beginning the entanglement disappears faster when the 'classical' part of the system is affected by the environment. Moreover, in this case the entanglement disappears completely and never revives. If the 'quantum' subsystem interacts with the environment, a single entanglement revival occurs.
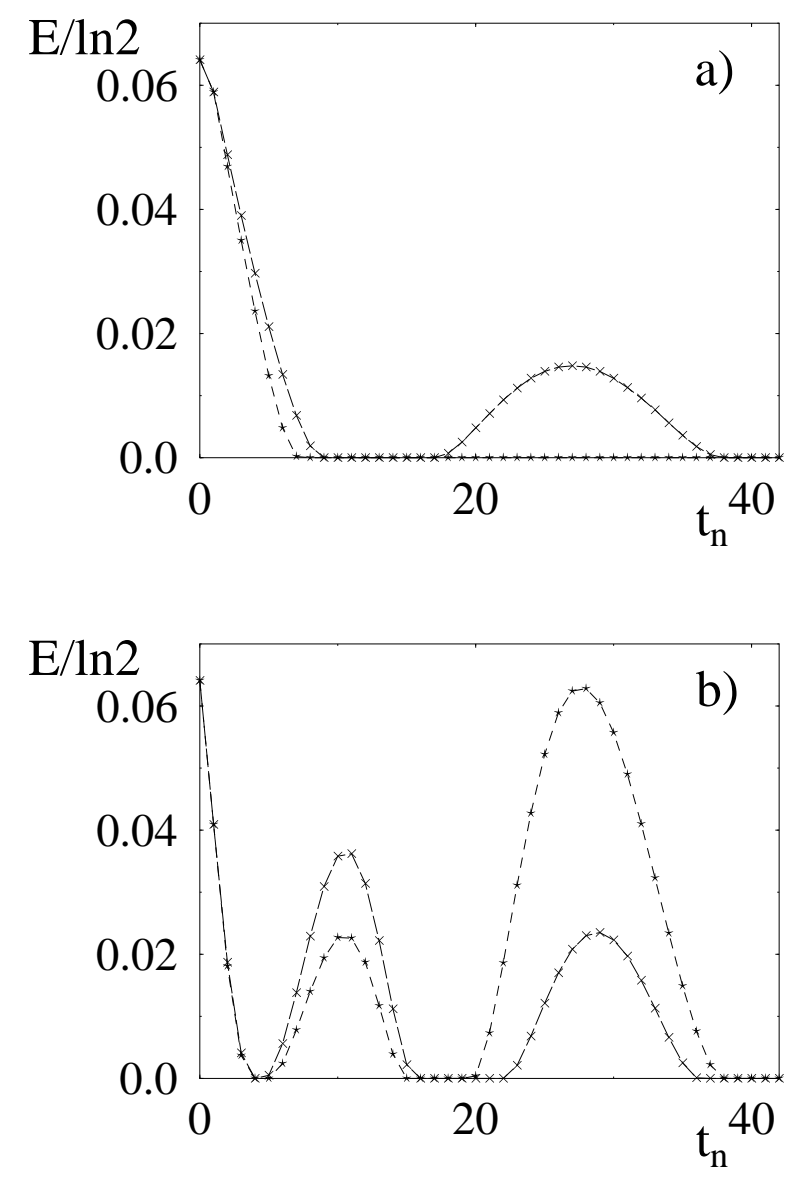

FIG. 10. As in Fig. 8 for $\varepsilon=0.01$ and $\alpha=0.04$.

In the complementary case, for which $\varrho^{(1)}$ interacts with the reflected Hamiltonian $H^{\prime}$ (see 10.b), we observe a special kind of competition: for short times the entanglement is smaller, if the quantum subsystem is per- turbed. For longer times, the roles are interchanged, and the oscillations of the entanglement are damped faster, if the classical subsystem interacts with the environment.

In general one can see that the pictures corresponding to the cases (a) and (b) are qualitatively different depending on the ratio $\epsilon / \alpha$. This fact may be related to the observation concerning the processes of decoherence. Depending on the relation between two coupling parameters the so called pointer basis is determined either by the internal self-Hamiltonian of the system or by the Hamiltonian of the interaction with environment [3].

\section{DISCUSSION}

We investigated the behaviour of entanglement of bipartite spin- $\frac{1}{2}$ system subjected to periodic action of the environment. The process of destruction of entanglement of initially pure states is accompanied by increasing of von Neumann entropy. The asymptotic value of the entropy depends on the form of the interaction with the environment. For strongly mixing bistochastic channels, (e.g. $\Theta^{2}$ and $\Theta^{3}$ ) the entropy achieves the maximal value $\ln 4$. If the decaying channel is involved, the entropy gets its maximum and then it monotonically decays to the asymptotic value, which reveals the initial entanglement of the system.

If the internal unitary evolution entangling the system is present, the decay of the entropy due to the decaying channel can be replaced by the process of mixing the state more and more. The general feature of the time evolution is that the entanglement decreases as the system becomes more mixed. This corresponds to the results recently obtained in 114,15, where it was shown that the mean entanglement of quantum states, averaged over a sample of mixed states with the same von Neumann entropy, decreases with the degree of mixing. The presence of the internal unitary evolution leads to the revivals of the entanglement and to suppression (or acceleration) of the entanglement decay.

Perhaps the most intriguing is the character of asymmetry of the time evolution of the entanglement. For some initial mixed states consisting of two non-equivalent subsystems, the entanglement decays faster, if the environment interacts with the 'classical' subsystem, which satisfies the entropic inequality. Many years ago Schrödinger considered entanglement of pure state as a property of having both subsystems less informative for the observer, than the composite system. Mixed states (11) considered here exhibit this property only with respect to one subsystem [19]. Our results show that the action of environment to the 'classical' subsystem is sometimes more harmful to the entanglement. In this case one can thus say that the quantum entanglement runs away faster through the classical door.

In the context of the above discussion some general questions emerge. Consider a quantum entangled state $\varrho$ 
with, say, $S\left(\varrho_{A}\right)>S\left(\varrho_{B}\right)$, not necessarily violating the inequality (9). Under which conditions the entanglement is less robust to action of the environment on this subsystem, for which the entropy of the reduced operator is smaller? How is it related to a possible violation of the von Neumann entropy inequality by subsystem $A$ ? What happens if instead of the inequalities (9,10) one applies the generalised $\alpha$-entropies inequalities $(18,19,21$, satisfied for classical systems? All these questions seem to be important for deeper understanding of the dynamics of quantum entanglement.

It would be also interesting to analyse the role of entropic asymmetric states like (11) in context of quantum communication. In fact these states have only one coherent information positive [22,23] (see also [24]). For the corresponding quantum channels this might imply an asymmetry in the transfer of quantum information with respect to its direction, $(A \rightarrow B$ or $B \rightarrow A)$.

Finally, the obtained results show that even the simplest bipartite systems may exhibit non-trivial properties form the point of view of the information theory. In this context it would be important to investigate further the dynamics of mixed entanglement, in particular, by taking into account the phenomenon of bound entanglement 25.

It is a pleasure to thank the European Science Foundation and the Newton Institute for a support during our stay in Cambridge, where this work has been initiated. K. $\dot{Z}$ is supported by Polish Committee for Scientific Research, contract No. 2 PO3B 072 19. M. P. R. H. are supported by Polish Committee for Scientific Research, contract No. 2 P03B 10316 and by European Community under the IST project EQUIP, contract No. IST-1999-11053.

\section{APPENDIX A: RANDOM PURE STATES}

In this appendix we present algorithms allowing one to generate random quantum states distributed uniformly at the entire space of pure states, the manifold of separable pure states and the space of maximally entangled pure states. We concentrate here on the simplest $2 \otimes 2$ problem, but the algorithms below can be easily generalised for higher dimensions.

\section{Generation of random pure states}

The set of pure states of a 4 dimensional Hilbert space forms a complex projective space $\mathbb{C} P^{3}$, on which a natural, unitarily invariant measure exist. To generate random pure states according to such a measure on this 6 dimensional space we take a vector of a random unitary matrix distributed according to the Haar measure on $U(4)$. The Hurwitz parametrization 26] gives

$$
\begin{array}{r}
|\Psi\rangle=\left(\cos \vartheta_{3}, \sin \vartheta_{3} \cos \vartheta_{2} e^{i \varphi_{3}},\right. \\
\left.\sin \vartheta_{3} \sin \vartheta_{2} \cos \vartheta_{1} e^{i \varphi_{2}}, \sin \vartheta_{3} \sin \vartheta_{2} \sin \vartheta_{1} e^{i \varphi_{1}}\right),
\end{array}
$$

where $\vartheta_{k} \in[0, \pi / 2]$, and $\varphi_{k} \in[0,2 \pi)$ for $k=1,2,3$.

A uniform distribution over almost all of $\mathbb{C} P^{3}$ is obtained by choosing the uniform distribution of the 'azimuthal' angles; $P\left(\varphi_{k}\right)=1 / 2 \pi$. In the analogy to the volume element on the sphere the 'polar' angles $\vartheta_{k}$ should be taken in a nonuniform way, with the probability density 26.

$$
P\left(\vartheta_{k}\right)=k \sin \left(2 \vartheta_{k}\right)\left(\sin \vartheta_{k}\right)^{2 k-2}
$$

for $\vartheta_{k} \in[0, \pi / 2], k=1,2,3$. In practice it is convenient to use auxiliary independent random variables $\xi_{k}$ distributed uniformly in $[0,1]$ and to set $\vartheta_{k}=\arcsin \left(\xi_{k}^{1 / 2 k}\right)$. Above formula with $k=1,2, \ldots, N-1$ allows one to get a natural distribution on $\mathbb{C} P^{N-1}$ 27].

\section{Random separable pure states}

Any $2 \otimes 2$ pure separable state may be written as $\left|\Psi_{s}\right\rangle=$ $\left|\psi_{1}\right\rangle \otimes\left|\psi_{2}\right\rangle$, where $\left|\psi_{1}\right\rangle$ and $\left|\psi_{2}\right\rangle$ are $N=2$, one-particle pure states. The 4 dimensional manifold of separable states has thus a simple structure of a Cartesian product $\mathbb{C} P^{1} \times \mathbb{C} P^{1}$. A uniform measure on this manifold is obtained be taking both states $\left|\psi_{i}\right\rangle$ distributed uniformly (and independently) at the Bloch sphere, $\mathbb{C} P^{1} \sim S^{2}$.

Working in the standard basis,

$$
\left|\Psi_{s}\right\rangle=U_{1} \otimes U_{2}|(1,0,0,0)\rangle,
$$

where $U_{1}$ and $U_{2}$ denote two independent random unitary matrices distributed uniformly on $S U(2)$. This parametrisation describes the entire $4 \mathrm{D}$ manifold of the separable pure states.

\section{Random maximally entangled states}

In an analogous way we may represent the maximally entangled states as

$$
\left|\Psi_{e}\right\rangle=\mathbb{I} \otimes U_{1}|(0,1,1,0) / \sqrt{2}\rangle .
$$

It is easy to see that for this states the reduced density matrix is proportional to identity matrix, and the entropy of entanglement achieves its maximum $\ln 2$. The states obtained by a symmetric operations $U_{1} \otimes \mathbb{I}$ are also maximally entangled. Using the standard representation of $U_{1}$ we parametrise maximally entangled states by 28]

$$
\left|\Psi_{e 1}\right\rangle=\frac{1}{\sqrt{2}}\left[\begin{array}{l}
\cos \vartheta e^{i \varphi_{1}} \\
\sin \vartheta e^{i \varphi_{2}} \\
-\sin \vartheta e^{-i \varphi_{2}} \\
\cos \vartheta e^{-i \varphi_{1}}
\end{array}\right] .
$$


The angles $\varphi_{i}$ are distributed uniformly in $[0,2 \pi)$, whereas according to (A1) $P(\vartheta)=\sin (2 \vartheta)$ for $\vartheta \in$ $[0, \pi / 2]$. Note that the standard element of the volume on the two sphere $d S=\sin \theta d \theta d \varphi$ is written in a rescaled variable $\theta=2 \vartheta$. Given maximally entangled state corresponds to a single unitary matrix $U_{1}$ pertaining to $S U(2)$, but the 3 -D manifold of the maximally entangled states has the topology of the real projective space, $\mathbb{R} P^{3} \sim U(3) / U(1)$ (20.

* Electronic address: karol@cft.edu.pl

** Electronic address: pawel@mifgate.pg.gda.pl

*** Electronic address: michalh@iftia.univ.gda.pl

¥ Electronic address: fizrh@univ.gda.pl

[1] A. Einstein, B. Podolsky and N. Rosen, Phys. Rev. 47, 777 (1935)

[2] E. Schrödinger, Proc. Cambridge Philos. Soc. 31, 555 (1935).

[3] W. H. Żurek, Phil. Trans. R. So. Lond. A 356, 1793 (1998).

[4] X.X. Yi and C.P. Sun, Phys. Lett. A 262, 282 (1999).

[5] K. Krauss, Ann. Phys., 64, 311 (1971), E. B. Davies, Quantum theory of open systems, Academic Press, London 1976.

[6] R. Alicki, K. Lendi, Quantum dynamical semigroups and applications, Springer-Verlag 1987.

[7] A. Łoziński and K. Życzkowski, to be published.

[8] P. Badzia̧g, M. Horodecki, P. Horodecki and R. Horodecki, Phys. Rev. A 62, 012311 (2000).

[9] See J. Preskill, at www.theory.caltech.edu/people/preskill/ph229.

[10] R. Penrose, The Emperor's New Mind, Oxford University Press, Oxford 1989.

[11] C. H. Bennett, D. P. DiVincenzo, J. Smolin and W. K. Wootters, Phys. Rev. A 54, 3814 (1997).

[12] R. Horodecki, P. Horodecki, Phys. Lett. A, 194, 147 (1994).

[13] S. Hill and W. K. Wootters, Phys. Rev. Lett. 78, 5022 (1997); W. K. Wootters, Phys. Rev. Lett. 80, 2245 (1998).

[14] K. Życzkowski, P. Horodecki, A. Sanpera, M. Lewenstein Phys. Rev. A 58, 833 (1998).

[15] K. Życzkowski, Phys. Rev. A 60, 3496 (1999).

[16] S. L. Braunstein, Phys. Lett. A 219, 169 (1996).

[17] M. J. W. Hall, Phys. Lett. A 242, 123 (1998).

[18] R. Horodecki, P. Horodecki and M. Horodecki, Phys. Lett. A 210, 377 (1996).

[19] R. Horodecki, Phys. Lett. A 210, 223 (1996).

[20] R. Horodecki and M. Horodecki, Phys. Rev. A 54, 1838 (1996).

[21] P. Horodecki, J. A. Smolin, B. M. Terhal, A. V. Thapliyal, LANL preprint quant-ph/9910122.

[22] B. Schumacher, Phys. Rev. A 54, 2614 (1996); B. Schumacher and M. A. Nielsen, Phys. Rev. A 54, 2629 (1996).

[23] S. Lloyd, Phys. Rev. A 56, 1613 (1997).

[24] M. Horodecki, P. Horodecki and R. Horodecki, Phys. Rev.
Lett. 85, 433 (2000).

[25] M. Horodecki, P. Horodecki and R. Horodecki, Phys. Rev. Lett. 80, 5239 (1998); P. Horodecki, M. Horodecki and R. Horodecki ibid 82, 1046 (1999).

[26] A. Hurwitz, Nachr. Ges. Wiss. Gött. Math.-phys. Kl. 71, (1897).

[27] M. Poźniak, K. Życzkowski, and M. Kuś, J. Phys. A 31, 1059 (1998).

[28] M. Kuś and K. Życzkowski, LANL preprint quantph/0006068 and Phys. Rev. A, (2001), in print.

[29] K. G. H. Vollbrecht and R.F. Werner, J. Math. Phys. 41, 6772 (2000). 


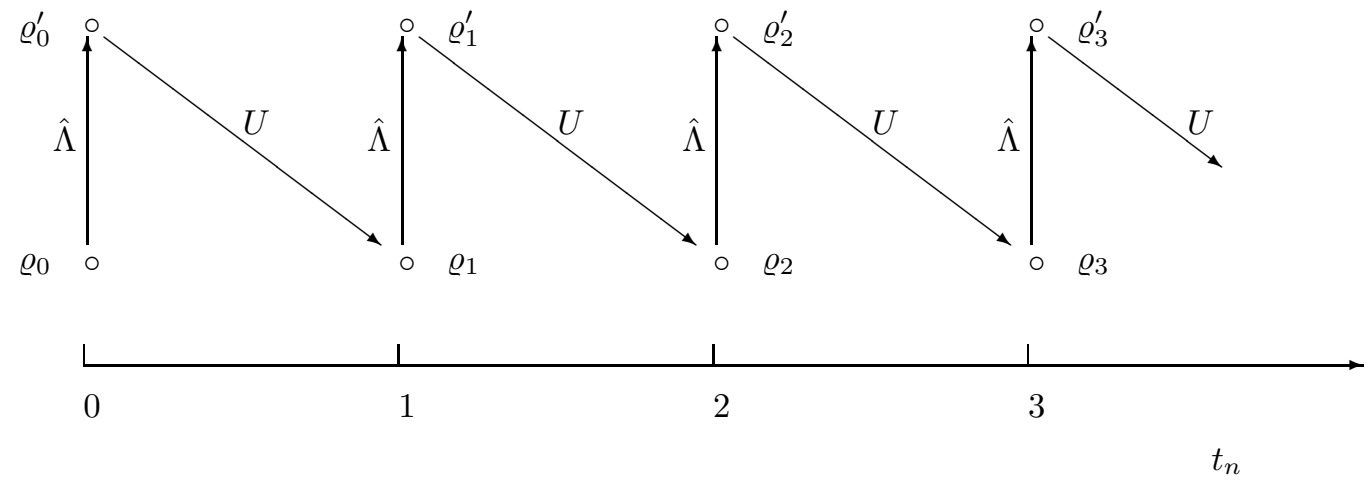

FIG. 1. Discrete model of periodic dynamics ( $($ ), (cf. Fig. $8.1,8.2$ of Ref. 10 ). Interaction with the environment $\hat{\Lambda}$ transforms the state $\varrho_{n}$ into $\varrho_{n}^{\prime}$ and then the unitary transformation $U$ maps it into $\varrho_{n+1}$. 